\title{
A study on the AC breakdown voltages of as-received palm oil and coconut oil under presence of $\mathrm{TiO} 2$
}

\begin{abstract}
This paper presents a study on the AC breakdown voltages of Palm Oil (PO) and Coconut Oil (CO) with presence of TiO2. The type of PO used in this study is Refined, Bleached and Deodorized Palm Oil (RBDPO). TiO2 was added into RBDPO and CO at volume concentration of nanoparticles ranging from $0.001 \%$ to $0.05 \%$ In total, 50 measurements of AC breakdown voltages were recorded and analyzed by Weibull and normal distributions. Based on the test results and statistical analyses, it was found that the $\mathrm{TiO} 2$ could improve the AC breakdown voltages of RDBPO and CO.
\end{abstract}

Keyword: AC breakdown voltage; Bleached and deodorized palm oil; Coconut oil; Dielectric insulating fluid; Refined; TiO2; Transformers 